\title{
EFFECT OF FLUORIDE-CONTAINING DESENSITIZING AGENTS ON THE BOND STRENGTH OF RESIN-BASED CEMENTS TO DENTIN
}

\author{
Duygu SARAÇ ${ }^{1}$, Safak KÜLÜNK ${ }^{2}$, Y. Sinasi SARAÇ ${ }^{2}$, Özlem KARAKAS ${ }^{3}$
}

1- DDS, PhD, Associate Professor, Department of Prosthetic Dentistry, Faculty of Dentistry, Ondokuz Mayls University, Samsun, Turkey.

2- DDS, PhD, Assistant Professor, Department of Prosthetic Dentistry, Faculty of Dentistry, Ondokuz Mayls University, Samsun, Turkey.

3- DDS, Research Assistant, Department of Prosthetic Dentistry, Faculty of Dentistry, Ondokuz Mayls University, Samsun, Turkey.

Corresponding address: Dr. Duygu SARAÇ - Ondokuz Mays University - Dental Faculty - Department of Prosthodontics - 55139 Kurupelit- SAMSUN - TURKEY - Phone: +90 3623121919 /3000 - Fax : +90 3624576032 - e-mail: dsarac@omu.edu.tr

Received: September 18, 2008 - Modification: November 11, 2008 - Accpeted: January 18, 2009

\begin{abstract}
$O$

bjective: The objective of this study was to evaluate the effect of desensitizing agents containing different amounts of fluoride on the shear bond strength of a dual polymerized resin cement and a resin-modified glass ionomer cement (RMGIC) to dentin. Material and Methods: One hundred human molars were mounted in acrylic resin blocks and prepared until the dentin surface was exposed. The specimens were treated with one of four desensitizing agents: Bifluorid 12, Fluoridin, Thermoline and PrepEze. The remaining 20 specimens served as untreated controls. All groups were further divided into 2 subgroups in which a dual polymerized resin cement (Bifix QM) or a resin-modified glass ionomer cement (AVANTO) was used. The shear bond strength (MPa) was measured using a universal testing machine at a $0.5 \mathrm{~mm} / \mathrm{min}$ crosshead speed. The data were analyzed statistically with a 2-way ANOVA, Tukey HSD test and regression analysis $(\alpha=0.05)$. The effect of the desensitizing agents on the dentin surface was examined by scanning electron microscopy. Results: The fluoride-containing desensitizing agents affected the bond strength of the resinbased cements to dentin $(\mathrm{p}<0.001)$. PrepEze showed the highest bond strength values in all groups $(\mathrm{p}<0.001)$. Conclusion: Regression analysis showed a reverse relation between bond strength values of resin cements to dentin and the amount of fluoride in the desensitizing agent $(\mathrm{p}<0.05)$.
\end{abstract}

Key words: Desensitizing agents. Fluorid. Resin cement.

\section{INTRODUCTION}

During conventional tooth preparation, approximately 1.2 to $1.5 \mathrm{~mm}$ of tooth structure is removed to ensure appropriate crown contours and adequate occlusal clearance ${ }^{28}$. After preparation, 23,000-35,000/ $\mathrm{mm}^{2}$ dentinal tubulus, 1 to $2 \mathrm{~mm}$ from the pulp and $19,000 \mathrm{~mm}^{2}$ dentinal tubules subjacent to the amelodentinal junction are exposed $\mathrm{d}^{33,34}$ and may cause dentinal hypersensitivity ${ }^{3,10,31}$. Brannström's hydrodynamic theory can explain the dentinal hypersensitivity ${ }^{5,6}$. This theory suggests that chemical, thermal, or osmotic stimuli cause the fluid within the tubules to flow inward or outward, creating a mechanical disturbance which can excite nerve fibers in the pulp and induce pain ${ }^{5,6}$.

There are several treatment options for managing dentinal hypersensitivity. Chemical or physical agents are used to either desensitize the nerve or to cover the exposed dentinal tubules ${ }^{3}$. Other treatments for the dentinal hypersensitivity involve occlusion of dentinal tubules, application of sedative agents, and promotion of dentin remineralization ${ }^{16,29}$. Several types of dentin desensitizers, such as varnishes, antiinflammatory agents, tubular obturating procedures, or dentin bonding agents and restorative resins may be applied to the tooth after cavity and crown preparation ${ }^{19,29,35}$. Desensitizing agents stimulate mineral deposition or occlude dentinal tubules to reduce dentinal hypersensitivity ${ }^{19}$. Adhesives or fluoride solutions may be applied to the exposed dentin surfaces to prevent dentinal hypersensitivity by sealing the open dentinal tubules ${ }^{4,9,12,25}$. In addition, primers containing glutaraldehyde and hydroxymethyl methacrylate (HEMA) can also reduce hypersensitivity by occluding dentinal tubules, possibly by precipitating plasma proteins in the dentinal fluid ${ }^{7,8}$.

Topical methods are widely used for dentinal hypersensitivity because of their convenience and immediate effect ${ }^{3,18,27}$. Topical desensitizers and dentifrices containing sodium fluoride, calcium fluoride, ferric, aluminum and potassium oxalates are the first choice treatments for dentinal hypersensitivty ${ }^{14,15,18,24,27,31}$. The desensitizing effects of fluoride occur when precipitated fluoride compounds mechanically block exposed dentinal tubules or fluoride within the tubules 
blocking transmission of the stimuli ${ }^{3}$.

As a treatment option, fluoride varnish forms globules of calcium fluoride both on the enamel and dentin surface, which act as reservoirs for releasing fluoride into the dentin tubules . As a result, this agent reduces dentinal hypersensitivity ${ }^{9}$, but decreases the bond strength of composite resin to dentin ${ }^{11,18}$.

Cementation is one of the reasons of hypersensitivity ${ }^{20}$. Cement may cause tooth sensitivity by the pressure created by cement before setting ${ }^{20}$. The cement can displace an equal amount of dentinal fluid, which may cause excessive hydrostatic pressure and resultant irritation of pulpal tissues ${ }^{20}$. Sealing of dentin with a desensitizing agent before cementation greatly decreases the hypersensitivity ${ }^{31}$. Desensitizing agents can be applied on prepared tooth surfaces to avoid complications during fabrication of restoration ${ }^{13,20}$. Various cements such as zinc phosphate, zinc polycarboxylate, conventional glassionomer cements (GICs), classified as acid-base cements, have been used for luting restorations ${ }^{2,23,33}$. A previous study showed that when GIC was used, sensitivity was frequent if the remaining dentinal thickness was less than one millimeter ${ }^{30}$. This sensitivity may be caused from the prolonged low $\mathrm{pH}$ of cement during setting and/or hydrostatic pressure that enabled the cement to enter dentinal tubules ${ }^{37}$. However, this information cannot explain the long lasting hypersensitivity reported by the patients.

In order to decrease the hypersensitivity, desensitizing agents are used before cementation. However it was reported that the use of desensitizing agents affects the bonding between dentin and luting material ${ }^{31}$. Durable bonding between dentin and luting materials in such cases is one of the most important factors for avoiding detachment of restorations as well as the prevention of microleakage, secondary dental caries and tooth fracture ${ }^{13,29}$. Some ingredients contained in dentin desensitizers may induce chemical interaction with dentin organic substances, and this may affect the sealing and bonding characteristics of the luting agents ${ }^{1,29}$.

This study aimed to evaluate the effect of 4 different desensitizing agents containing different amounts of fluoride on the shear bond strength of a dual polymerized resin cement and a RMGIC to dentin. Furthermore, dentin surfaces after the application of the desensitizing agents were examined under a field emission scanning electron microscope (SEM). The research hypothesis of this study was that the increase in the amount of fluoride in desensitizing agents could reduce the bond strength of resin cements to dentin.

\section{MATERIAL AND METHODS}

The bond strength test was performed using 50 unrestored and noncarious human third molars extracted due to periodontal reasons. Teeth were cleaned mechanically and stored in $0.5 \%$ chloramine at room temperature until use. Teeth were sectioned at the cementoenamel junction and the coronal parts were separated mesiodistally at the central groove with a water-cooled diamond-coated disc (910D; Diatech, Goltene AG, Altstatten, Switzerland). Specimens were then mounted, with the buccal or lingual surfaces facing upwards, in plastic holders containing autopolymerizing resin (Meliodent; Heraeus Kulzer Ltd, Armonk, NY, USA). The buccal and lingual surfaces were prepared with a standard-grit diamond rotary cutting instrument (105-125 $\mu \mathrm{m}$, Diatech) until the dentin surface was exposed, then the dentin surface preparation was finished with a finegrit diamond rotary cutting instrument ( $45 \mu \mathrm{m}$, Diatech).

The specimens were divided into 5 groups each containing 20 specimens. The dentin surfaces on control group had no surface treatment, remaining four groups were treated with Bifluorid 12, Fluoridin, Thermoline and PrepEze desensitizing agent according to manufacturer instructions, respectively. Desensitizing agents were applied to dentin surfaces with a cotton pellet. All desensitizing agents used in this study are presented in Table 1. All procedures were performed by the same investigator (S.K).

After the application of desensitizing agents, all groups were divided further into 2 subgroups $(n=10)$ in which a dual polymerized resin cement (Bifix QM) or a RMGIC (Avanto) were applied to the specimens according to manufacturer's instructions. A transparent plastic mold with a hole $(6 \mathrm{~mm}$ diameter and $2 \mathrm{~mm}$ height) in the center was used to place the cements on the dentin surface. Before application of RMGIC, dentin surfaces were etched with a $35 \%$ phosphoric acid gel (Vococid; VOCO America, Inc., New York, NY, USA) for 15 $\mathrm{s}$, rinsed with distilled water, and air dried. Two components of Avanto primer (primers A and B) were mixed for $5 \mathrm{~s}$ in a ratio 1:1 and applied to dentin surfaces for $30 \mathrm{~s}$. Avanto cement liquid and powder was mixed in a ratio 1:1 and was placed into the mold on dentin surface. For dual polymerized resin cement, dentin surfaces of the specimens were acid etched (Vococid; VOCO America, Inc.) for $15 \mathrm{~s}$, rinsed and dried. The primer (Solobond Plus Primer) and the adhesive (Solobond Plus Adhesive) were applied to the dentin surfaces for 30 and $15 \mathrm{~s}$, respectively. Subsequently, the adhesive was polymerized for $20 \mathrm{~s}$ with a light-curing unit with an intensity of $600 \mathrm{~mW} / \mathrm{cm}^{2}$

TABLE 1- Desensitizing agents

\begin{tabular}{llcl}
\hline Material & \multicolumn{1}{c}{ Contents } & Batch Number & Manufacturer \\
\hline Bifluorid 12 & $2.71 \%$ sodium fluorid & 360315 & VOCO, Cuxhaven, Germany \\
& $2.92 \%$ calcium fluorid & & \\
Fluoridin & $2.26 \%$ sodium fluorid & 480356 & VOCO, Cuxhaven, Germany \\
Thermoline & $1 \%$ sodium fluorid & 490261 & VOCO, Cuxhaven, Germany \\
PrepEze & $1 \%$ calcium fluorid & & \\
\hline
\end{tabular}


(Astralis 3, Ivoclar, Vivadent, Schaan, Liechtenstein). The resin cement was placed into the mold and polymerized for $20 \mathrm{~s}$.

The specimens were stored in distilled water at $37^{\circ} \mathrm{C}$ for $24 \mathrm{~h}$ before testing. After storage, shear bond strengths were measured in a universal testing machine (Lloyd LRX; Lloyd Instruments PIC., Fareham, Hampshire, England) at a crosshead speed of $0.5 \mathrm{~mm} / \mathrm{min}$. Data were analyzed by 2 -way analysis of variance (SPSS 12.0; SPSS Inc., Chicago, IL, USA). Means and standard deviation of bond strengths were calculated and mean values were compared by the Tukey HSD test $(\alpha=0.05)$. Then, to evaluate the relation between the bond strength and the amount of fluoride of desensitizing agents a regression analysis was performed $(\alpha=0.05)$ (SPSS 12.0; SPSS Inc.).

TABLE 2- Shear bond strength (MPa) for resin cement subgroups

\begin{tabular}{lll}
\hline Groups & Subgroups & Mean \pm SD \\
\hline \multirow{2}{*}{ Control } & Bifix QM & $4.90(0.14)$ \\
& Avanto & $3.69(0.20)^{\mathrm{a}}$ \\
Bifluorid 12 & Bifix QM & $2.65(0.19)^{\mathrm{b}, \mathrm{c}}$ \\
& Avanto & $2.27(0.16)^{\mathrm{b}, \mathrm{c}}$ \\
Fluoridin & Bifix QM & $2.70(0.15)^{\mathrm{b}}$ \\
& Avanto & $2.15(0.15)^{\mathrm{c}}$ \\
Thermoline & Bifix QM & $3.91(0.27)$ \\
& Avanto & $3.26(0.10)^{\mathrm{a}}$ \\
Prepeze & Bifix QM & $8.09(0.68)$ \\
& Avanto & $4.79(0.10)$ \\
\hline
\end{tabular}

Same letters indicate statistically significant difference at $5 \%$ significnace level
To evaluate the dentin surface of the specimens after application of the desensitizing agents, for each group one tooth, totally 5 additional teeth were prepared and the desensitizing agents were applied to them. Acid etching was not applied to these specimens. Subsequently, specimens were gold sputtered with a sputter coater (S150B; Edwards, Crawley, England) and examined by a field emission SEM (JSM-6335F; JEOL Ltd, Tokyo, Japan) at 15.0 or $20.0 \mathrm{kV}$. The SEM photomicrographs were developed with $\times 2,000$ magnification for visual inspection. In addition, a stereomicroscope (Stemi 2000-C; Carl Zeiss, Gottingen, Germany) at a magnification of $\times 10$ was used to evaluate the type of failure. The nature of the failure was noted as adhesive, cohesive, or mixed.

\section{RESULTS}

The results showed that the type of desensitizer agent, cement and their interactions were statistically significant $(p<0.05)$. The type of desensitizer affected the shear bond strength of resin cements to dentin significantly $(p<0.05)$. For all desensitizer groups, the dual polymerized adhesive resin cement showed higher bond strength values than the RMGIC $(p<0.05)$ except Bifluoride 12 groups $(p>0.05)$. Application of Bifix QM after dentin surfaces treated with PrepEze showed the higher shear bond strength values (8.09) than the other groups $(p<0.05)$. There were no significant differences between application of Bifluorid 12 and Fluoridin cemented with either the dual polymerized adhesive resin cement or the RMGIC (p>0.05) (Table 2).

Regression analysis showed a reverse relation between bond strength values of resin cements to dentin and the amount of fluoride in the desensitizing agent $(\mathrm{R} 2=0.70, \mathrm{p}<0.05)$

Mixed and adhesive fractures were the most common failure

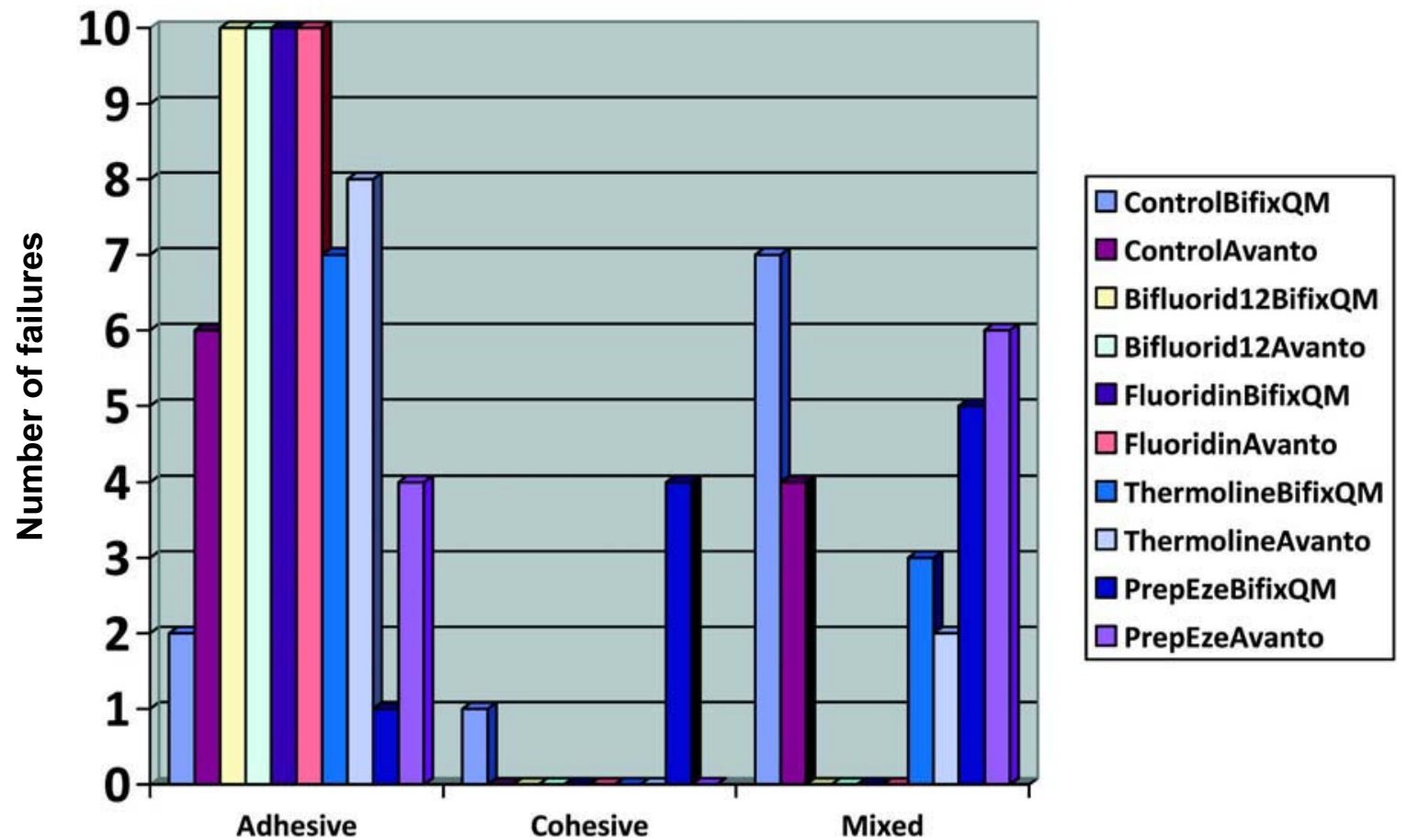

FIGURE 1- Failure modes of the bonding between cements and dentin according to the applied desensitizing agents $(n=10)$ 

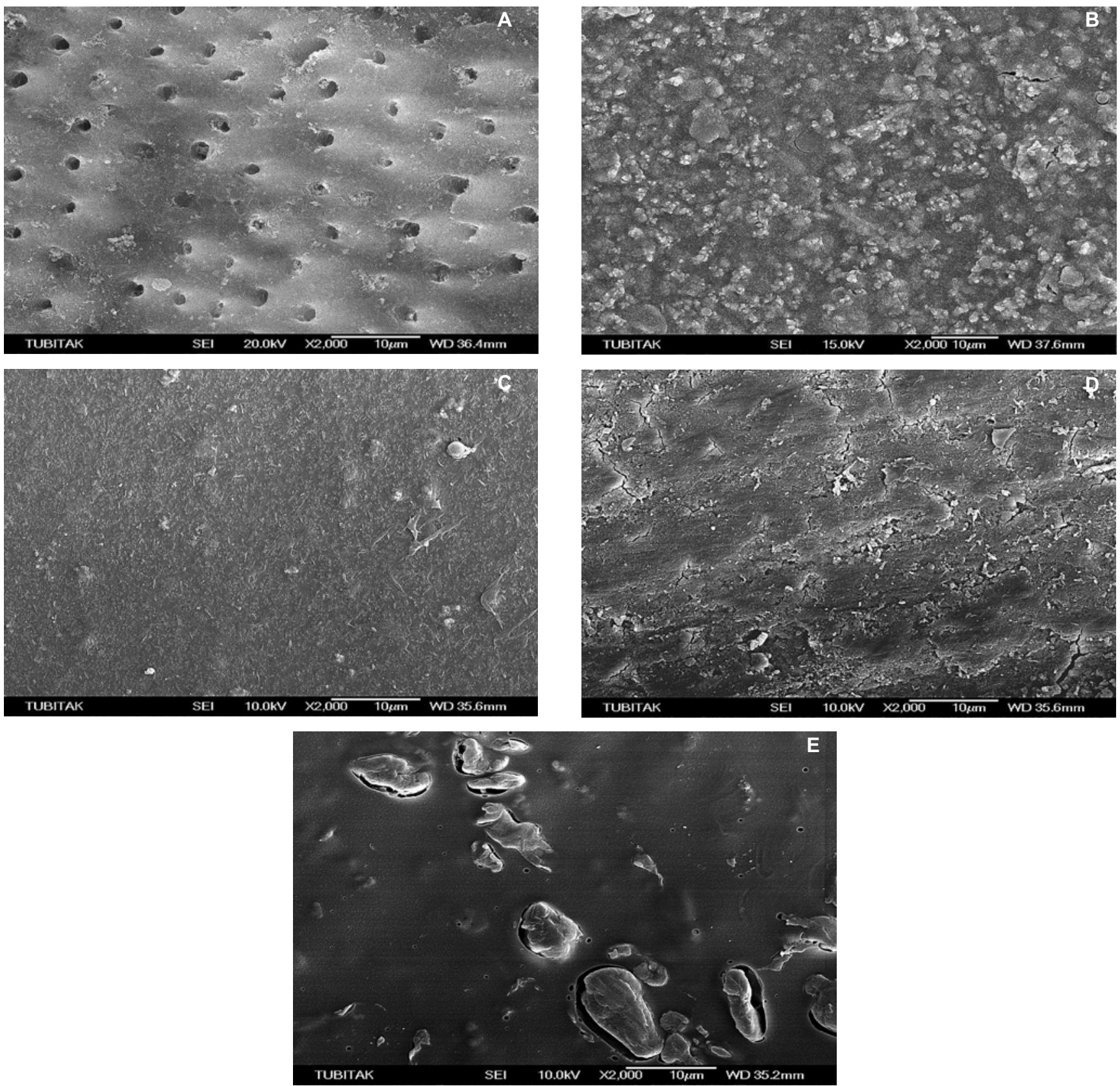

FIGURE 2- SEM micrographs of dentin surfaces of specimens treated with the desensitizing agents. A, control (no treatment); $\mathrm{B}$, treated with $2.71 \% \mathrm{NaF}$ and $2.92 \% \mathrm{CaF}$-containing desensitizing agent (Bifluorid 12); C, treated with $2.26 \% \mathrm{NaF}-$ containing desensitizing agent (Fluoridin); D, treated with $0.5 \% \mathrm{NaF}$ and $35 \%$ HEMA-containing desensitizin agent (PrepEze); E, treated with $1 \% \mathrm{NaF}$ and $1 \% \mathrm{CaF}$-containing desensitizing agent (Thermoline). Original magnification $\times 2000$

types for the groups. While mixed failures were seen with the use of dual polymerized resin cement (70\%), adhesive failures were obtained with the resin modified glass ionemer cement $(60 \%)$ in the control groups. The specimens treated with Bifluorid 12 or Fluoridin showed $100 \%$ of adhesive failures. Cohesive failures were obtained only with the use of dual polymerized resin cement in PrepEze (40\%) and control groups (10\%) (Figure 1).

Exposed dentin tubules can be seen clearly in the control specimen (Figure 2,A). SEM evaluation revealed that the dentin tubules were covered with desensitizing agents (Figure 2, BE). Fluoride precipitants were seen on the dentin surface on
Figure 2, B and C.

\section{DISCUSSION}

It was hypothesized that 4 different desensitizing agents with different amount of fluoride would affect the bond strength of the 2 different resin cements to dentin. The hypothesis is verified by the result of this study. The increase in the amount of fluoride of desensitizing agents decreased the bond strength of resin cements to dentin. While HEMA and $0.5 \%$ sodium fluoride-containing desensitizing agent 
(PrepEze) demonstrated higher bond strengths, agents with higher amount of fluoride demonstrated lower bond strength values than control specimens. This reduction may result from crystal precipitation ${ }^{9,11,26,29}$ during application of fluoridecontaining agents. These crystals are acid-resistant and may chemically and physically prevent complete penetration of the resin components of the dual polymerized adhesive resin cement and RMGICs. Some investigations ${ }^{12,13,29}$ have shown that fluoride-containing agents demonstrated lower bond strength values to sound dentin than HEMA-containing desensitizing agents ${ }^{12,13,29}$. Kolker, et al. ${ }^{16}$ evaluated the effect of desensitizing agents categorized by their proposed mechanism for decreasing the fluid flow in the dentinal tubules on dentin permeability by resin occlusion, precipitation of proteins and precipitation of crystals. Dentin desensitizing agents tested showed a wide range of ability in reducing dentin permeability and the agents that precipitate crystals into tubules were more effective than others ${ }^{16}$.

The results of the present study show that HEMAcontaining desensitizing agent (PrepEze) increased the bond strength of resin cements. HEMA, as an example of a hydrophilic primer, is used to improve the infiltration of adhesive monomers into demineralized dentin by wetting the surface of collagen fibers and maintains the collagen network in an expanded state by stiffening the collagen fibers ${ }^{12,13}$. In addition, the increase in the bond strength of the group treated with HEMA-containing desensitizing agent can be attributed to the polymerization of HEMA leaving of a film of polymerized material on dentin surface ${ }^{21,22}$. Also, HEMA increases the infusion and impregnation of resin monomers into the demineralized dentinal matrix. Thus, the interfacial hybrid zone formed by HEMA must have played an important role in enhancing the bonding of the resin materials ${ }^{17}$.

Based on the results of the present study, it is suggested that the amount of fluoride affects the shear bond strength of the cements. Higher fluoride amounts resulted in lower bond strengths. The higher amount of fluoride covers more dentin surface as seen in SEM photomicrographs. Although dentin tubules were occluded completely in the image of the specimen treated with HEMA and $0.5 \%$ fluoride-containing agent (PrepEze) (Figure 2D), this group showed better bond strength than the other experimental groups. This result can be attributed to PrepEze being a resin-based desensitizing agent. It is thought that an increase in precipitated crystals on dentin surface with higher amount of fluoride results in the weaker bond strength. Wolfart, et al. ${ }^{35}$ evaluated the effect of on one low filled, one highly filled resin sealers and one HEMA-containing desensitizing agent on shear bond strength of conventional glass ionomer cement to dentin by comparing with calcium hydroxide suspension. Those authors ${ }^{35}$ reported that a low filled resin sealer and a HEMA-containing desensitizing agent did not show any differences in bond strength compared to the standard desensitizing method using calcium hydroxide suspension. The use of highly filled resin sealer had a significantly negative effect on bonding when compared to control. In another study ${ }^{32}$, the effect of enamel etchant and a desensitizer (PrepEze) on the bond strength of orthodontic resin to enamel. PrepEze significantly decreased the bond strength of the resin, which is contrary to the findings of the present study ${ }^{32}$. This difference can be attributed to the treated surfaces. While those authors ${ }^{35}$ used enamel surfaces in the present study dentin surfaces were used to simulate the prepared tooth surfaces for fixed partial dentures.

In this study, all desensitizing agents showed lower bond strength values with RMGIC $(\mathrm{p}<0.05)$. For the RMGIC, adhesion is probably achieved by a combination of chemical bonding, which occurs between carboxylic acid $(\mathrm{COOH})$ group of cement and calcium ions of dentin, and the micromechanical bonding mechanism described for resinbased adhesives ${ }^{2,36}$. The better performance of RMGICs compared to conventional GICs could be due to their expected dual mechanism of adhesion ${ }^{2}$. Resin cements are composites of a resin matrix, such as bis-GMA or urethane dimethacrylate, and fine inorganic particles as filler ${ }^{33}$. Because of their successful use in the cementation of resin bonded fixed partial dentures, the popularity of these materials has increased recently for crown cementation because of their use in conjunction with dentin bonding agents. Also, their mechanical and physical properties are better than that of other cements and their bond strength to dentin is higher than that of $\mathrm{RMGICs}^{23,33}$

When the failure modes were investigated, groups in which resin cement was used showed mixed failure. Also, in the group in which resin cement was used preceding the HEMAcontaining desensitizing agent, cohesive failure was observed, which were considered when more than $50 \%$ of the adhesive cement remained on dentin surfaces, implying that their interface is stronger than the material's cohesive strength. Therefore, the interfacial strength is greater that the recorded numerical data.

The $\mathrm{pH}$ value of the desensitizing agents may play a role on the bond strength of cements because of the effect on the solubility parameters of the materials. However, the $\mathrm{pH}$ of the desensitizing agents, except for Bifluoride 12 (pH: 5.6), were informed as unknown by the manufacturers.

In the present study, the highest bond strength values were obtained with HEMA-containing desensitizing agents. According to this result, it may be inferred that the bond strength of resin cements to dentin is related not only with the amount of fluoride, but also with the resin content of the material.

In the present study, only 1 type of adhesive resin cement and RMGIC and 4 types of desensitizing agents containing different amounts of fluoride were used and tests were done under in vitro conditions. Different results might be obtained with different experimental conditions, such as aging or fatiguing of specimens. Further research is necessary to evaluate the effects of the desensitizing agents.

\section{CONCLUSIONS}

Within the limitations of this study, the following conclusions were drawn:

1- All desensitizing agents used in this study reduced the bond strength of resin cements to dentin, except for the 
HEMA-containing desensitizing agent (Prepeze).

2- The HEMA-containing desensitizing agent showed higher bond strength values then control group.

3- The bond strength value of the resin cements to dentin showed an inverse relationship with the amount of fluoride of the desensitizing agents.

\section{REFERENCES}

1- Al Qahtani MQ, Platt JA, Moore BK, Cochran MA. The effect on shear bond strength of rewetting dry dentin with two desensitizers. Oper Dent 2003;28:287-96

2- Almuammar MF, Schulman A, Salama FS. Shear bond strength of six restorative materials. J Clin Pediatr Dent. 2001;25:221-5.

3- Bartold PM. Dentinal hypersensitivity: a review. Aust Dent J. 2006;51:212-8.

4- Bouillaguet $\mathrm{S}$, Wataha JC. Future directions in bonding resins to the dentin-pulp complex. J Oral Rehabil. 2004 31;385-92.

5- Brannstrom M. The cause of postrestorative sensitivity and its prevention. Endod. 1986;12:475-81.

6- Brannstrom M. The hydrodynamic theory of dentinal pain: sensation in preparations, caries, and the dentinal crack syndrome. J Endod. 1986;12:4537.

7- Dondi dall'Orologio G, Malferrari S. Desensitizing effects of Gluma and Gluma 2000 on hypersensitive dentin. Am J Dent. 1993;6:283-6.

8- Felton DA, Bergenholtz G, Kanoy BE. Evaluation of the desensitizing effect of gluma dentin bond on teeth prepared for complete-coverage restorations. Int J Prosthodont. 1991;4:292-8.

9- Gaffar A. Treating hypersensitivity with fluoride varnishes. Compend Contin Educ Dent. 1998;19:1088-90.

10- Holland G R, Narhi M N, Addy M, Gangarosa L, Orchardson R. Guidelines for the design and conduct of clinical trials on dentin hypersensitivity. J Clin Periodontol. 1997;24:808-13.

11- Itota T, Torii Y, Nakabo S, Yoshiyama M. Effect of fluoride application on tensile bond strength of self-etching adhesive systems to demineralized dentin. J Prosthet Dent. 2002;88:503-10.

12- Ivanyi I, Balogh AE, Fazekas A, Rosivall L, Nyarasdy I. Comparative analysis of pulpal circulatory reaction to an acetone containing and an acetone free bonding agent as measured by vitalmicroscopy. Oper Dent. 2002;27:367-72.

13- Ivanyi I, Balogh AE, Rosivall L, Nyarasdy I. In vivo examination of the scotchbond multi-purpose dental adhesive system in rat (vitalmicroscopic study). Oper Dent. 2000,25;418-23.

14- Jacobsen P L, Bruce G. Clinical dentin hypersensitivity: understanding the causes and prescribing a treatment. J Contemp Dent Pract. 2001;2:112 .

15- Jain P, Vargas MA, Denehy G E, Boyer DB. Dentin desensitizing agents: SEM and X-ray microanalysis assessment. Am J Dent. 1997;10:21-6.

16- Kolker JL, Vargas MA, Armstrong SR, Dawson DV. Effect of desensitizing agents on dentin permeability and dentin tubule occlusion. J Adhes Dent. 2002;4:211-21.

17- Kuo SM, Liou CC, Chuag CL, Wang YJ. Studies of the effects of PVAAE on dentinal bonding of HEMA. J Med Biol Eng. 2001;21:243-8.
18- Lehmann N, Degrange M. Effect of four dentin desensitizer on the shear bond strength of three bonding systems. Eur Cell Mater. 2002;9:52-

19- Ling TY, Gillam DG, Barber PM, Mordan NJ, Critchell J. An investigation of potential desensitizing agents in the dentin disc model: a scanning electron microscopy study. J Oral Rehabil. 1997;24:191-203.

20- Mausner IK, Goldstein GR, Georgescu M. Effect of two dentinal desensitizing agents on retention of complete cast coping using four cements. J Prosthet Dent. 1996;75:129-34.

21- Munksgaard EC, Asmussen E. Bond strength between dentin and restorative resins mediated by mixtures of HEMA and glutaraldehyde. J Dent Res. 1984;63:1087-9.

22- Munksgaard EC, Irie M, Asmussen E. Dentin-polymer bond promoted by Gluma and various resins. J Dent Res. 1985;64:1409-11.

23- Owens SE, Miller BH. A comparison of shear bond strengths of three visible light-cured orthodontic adhesives. Angle Orthod. 2000;70:352-6.

24- Pashley DH, Carvalho RM, Pereira JC, Villanueva R, Tay FR. The use of oxalate to reduce dentin permeability under adhesive restorations. Am $\mathrm{J}$ Dent. 2001;14:89-94

25- Pereira JC, Martineli AC, Tung MS. Replica of human dentin treated with different desensitizing agents: a methodological SEM study in vitro. Braz Dent J. 2002;13:75-85.

26- Schupbach P, Lutz F, Finger WJ. Closing of dentinal tubules by Gluma desensitizer. Eur J Oral Sci. 1997;105:414-21.

27- Seara SF, Erthal BS, Ribeiro M, Kroll L, Pereira GD. The influence of a dentin desensitizer on the microtensile bond strength of two bonding systems. Oper Dent. 2002;27:154-60.

28- Shillingburg HT, Hobo S, Whitsett LD. Fundamentals of fixed posthodontics. Chicago: Quintessence; 1981.

29- Soeno K, Taira Y, Matsumura H, Atsuta M. Effect of desensitizers on bond strength of adhesive luting agents to dentin. J Oral Rehabil. 2001;28:1122-8

30- Stanley HR. Local and systemic responses to dental composites and glass ionomers. Adv Dent Res. 1992;6:55-64.

31- Swift EJ Jr, Lloyd AH, Felton DA. The effect of resin desensitizing agents on crown retention. J Am Dent Assoc. 1997;128:195-200.

32- Vicente A, Bravo LA. Influence of an etchant and a desensitizer containing benzalkonium chloride on shear bond strength of brackets. J Adhes Dent. 2008;10(3):205-9.

33- Wassell RW, Barker D, Steele JG. Crowns and other extra-coronal restorations: try-in and cementation of crowns. Br Dent J. 2002;193:17-28.

34- Wilson NHF, Roulet JF, Fuzzi M. Advances in operative dentistry, challenges of the future. Chicago: Quintessence; 2001. v. 2.

35- Wolfart S, Linnemann J, Kern M. Crown retention with use of different sealing systems on prepared dentine. J Oral Rehabil. 2003;30:1053.

36- Yoshioka M, Yoshida Y, Inoue S, Lambrechts P, Vanherle G, Nomura Y, et al. Adhesion/decalcification mechanisms of acid interactions with human hard tissues. J Biomed Mater Res. 2002;59:56-62.

37- Zaimoglu A, Aydin AK. An evaluation of smear layer with various desensitizing agents after tooth preparation. J Prosthet Dent. 1992;68:4507. 